\title{
Classification of healthy and white root disease infected rubber trees based on relative permittivity and capacitance input properties using LM and SCG artificial neural network
}

\author{
Mohd Suhaimi Sulaiman, Zuraidi Saad
}

Faculty of Electrical Engineering, Universiti Teknologi MARA, Malaysia

\begin{tabular}{l}
\hline \hline Article Info \\
\hline Article history: \\
Received Nov 9, 2019 \\
Revised Jan 20, 2020 \\
Accepted Feb 2, 2020 \\
\end{tabular}

Keywords:

Artificial neural network

Classification

Levenberg marquardt

Scaled conjugate gradient

White root disease

\begin{abstract}
White root disease is one of the most serious diseases in rubber plantation in Malaysia that originally infects on the root surface of the rubber tree. So, prevention is important compared to treatment. The classification system proposed in the research had the ability of detecting the disease by classifying between healthy rubber trees and white root disease infected rubber trees. 600 samples of latex from healthy rubber trees and white root disease infected rubber trees were taken from the RRIM station in Kota Tinggi, Johor. These samples were measured based on its relative permittivity and capacitance. All of the measurement inputs from the experiment were tested using statistical analysis. These measurement input were then went through the process of classification in ANN to generate the optimized models by using LM and SCG algorithm. There were four optimized models selected from the classification process. The accuracy from the selected most optimized models were greater than $70 \%$. The selected most optimized models were then used to classify between healthy trees and white root infected trees based on single input categories.
\end{abstract}

\section{Corresponding Author:}

Mohd Suhaimi Sulaiman,

Faculty of Electrical Engineering,

Universiti Teknologi MARA,

Cawangan Pulau Pinang, Kampus Permatang Pauh, 13500, Pulau Pinang, Malaysia.

Email: suhaimisulaiman2009@gmail.com

\section{INTRODUCTION}

Artificial Neural Network (ANN) is a model of Artificial intelligence system inspired from astonishing human and animal biological brain work to make a decision and providing utilisation and understanding of potential function between human and artificial information processing system [1]. The behaviour of human brain starts from a learning process. The brain will process the input that came from the sensory organs. The same goes to artificial neural network, the intelligence system involving three stages starts from training, validation and testing. The interconnection between neurons, also known as hidden layer, is an important part to show the complexity of the system. In addition, the weight of every layer will change due to the training numbers and it will make the system increase in accuracy of making a decision. Nowadays, ANN has been used widely in engineering disciplines such as in electrical and computer engineering. The inputs obtained were processed through the structure which emulates the human brain performance [2]. The implementation of ANN can be seen in many applications such as (i) identification and recognition of complex dynamics, (ii) instrument and sensors calibration, and also in (iii) analog and digital signal processing [3]. 
Several works have been done through ANN to measure the permittivity of some materials. P. G. Bartley et al. [4] has trained ANN to determine the dielectric properties of eleven samples of water and isopropyl alcohol solutions. The coefficient of determination obtained was 0.999 . He used the method of feed forward and back propagation method. In other works that have been done, microwave technology research used ANN as a computational standard. C. Qian et al. [5] used ANN to measure the permittivity of material under microwave radiation. The permittivity of every sample was measured by using open ended coaxial probes. The data collected has become input to the ANN that uses Back Propagation (BP) as an algorithm. It was said that the $\mathrm{BP}$ algorithm can give the results in seconds and it is convenient to measure the permittivity of material even though the error is bigger compared with A. Hasan research [6]. A. Hasan [6] have measured the complex permittivity of materials using monopole antenna probe with the frequency range between 2.5-5 GHz. The probe immersed in the dielectric medium to measure the dielectric properties. After the data is obtained, the ANN algorithm was developed and Levenberg-Marquardt (LM) technique was used as a propagation algorithm. The result shows that error does not exceed $1 \%$ compared with the actual value.

The other researcher that used LM algorithm was done by H. Hadzli et al. [7] in classifying the rubber seed clones through imaging technique. He used 160 samples for training and 100 samples for testing part. The result shows that the performance achieved was $84.0 \%$ which can be considered as a good result. Other than that, there are other studies that used both LM and SCG for their research work. Prerana and Parveen Sehgal [8] had made a comparative study of Gradient Descent (GD), Levenberg-Marquardt (LM) and Scaled Conjugate Gradient (SCG) method of neural network for thyroid disease diagnosis. In their study, it had been observed that Levenberg Marquardt (LM) and Scale Conjugate Gradient (SCG) methods have shown a better training performance for achieving the set target in 31 epochs for (LM) and 56 epochs for (SCG) compared to Gradient Descent which require 1000 epochs. For classification, Sheeja Agustin [9] had compared between (LM) and (SCG) for classifying between normal and abnormal thyroid images. Both techniques have shown the performance accuracy more than $70 \%$. Based on the literature review, it shows that (LM) and (SCG) learning algorithm are among the best methods for classification process in ANN and is suitable to be implemented in the research for developing the classification model.

\section{METHODOLOGY}

ANN is a problem-solving tool that has become an alternative modelling method to systems with scientific or mathematical basis. ANNs have gained much attention as significant soft computing tools, not limited to data processing and analysis but can also be applied to solve difficulties in processes [10, 11]. The objective of the application of ANN in this research is to classify which tree is healthy and tree that has been infected with white root disease based on their measurement properties value. ANN classification was implemented with the use of LM and SCG algorithm in the research. Multilayer Perceptron with Back Propagation (MLP-BP) are the main algorithm in this research. A comparative method of LM algorithm (trainlm) versus SCG algorithm (trainscg) for data classifications was also presented to verify the effectiveness of the proposed algorithms. ANN Classification is the process of learning to separate samples into different classes, which are training and testing by finding common features between samples of known classes, which in this research is to find the difference between healthy and infected trees [12]. The best or most optimised model was also identified in the classification model by comparing between LM and SCG algorithm. Figure 1 shows the flowchart of the ANN modelling starting from data arrangement to classification system.

ANN Classification uses two architectures namely Multilayer Perceptron (MLP) and back propagation (BP). An MLP is a feed forward ANN, which maps sets of input neurons onto a set computational output [13]. By knowing the derivative of the function, back propagation algorithm could be beneficial for dividing the contribution of each weight by using three or more hidden layers with nonlinear activation function. It is also known to be the most widely applied learning algorithm for MLP in neural networks and employs gradient descent to minimise the squared error between the network output value and desired output value.

LM and SCG algorithms were part of the algorithm contained in the backpropagation category, which is a common method of training in ANNs [14]. In this research, examinations of two popular training algorithms (LM and SCG) were presented for detecting infected rubber trees with white root disease using the MLP classifier. The performance of the training algorithms was tested and compared on all of the experiment measurement data, which consisted of relative permittivity measurement and capacitance measurement. 
The datasets of the input measurement from all experiments were arranged neatly for ANN programming. The total number of 600 samples of natural rubber latex was divided into 300 samples from healthy rubber trees and 300 samples from rubber trees infected with white root disease. The system proposed had one inputs and one output, in which the results were either healthy or infected. These data from each measurement were organised to make it in the order of row and column in Excel. For ANN programming, the datasets were split into training, testing, and validation. From 600 samples, $70 \%$ will be used as training input and the remaining $30 \%$ was for testing and validation input. This would show that 420 datasets were taken for training, 90 datasets for testing, and another 90 datasets for validation. Breakdown of the datasets portion used in training, testing, and validation are illustrated in Table 1.

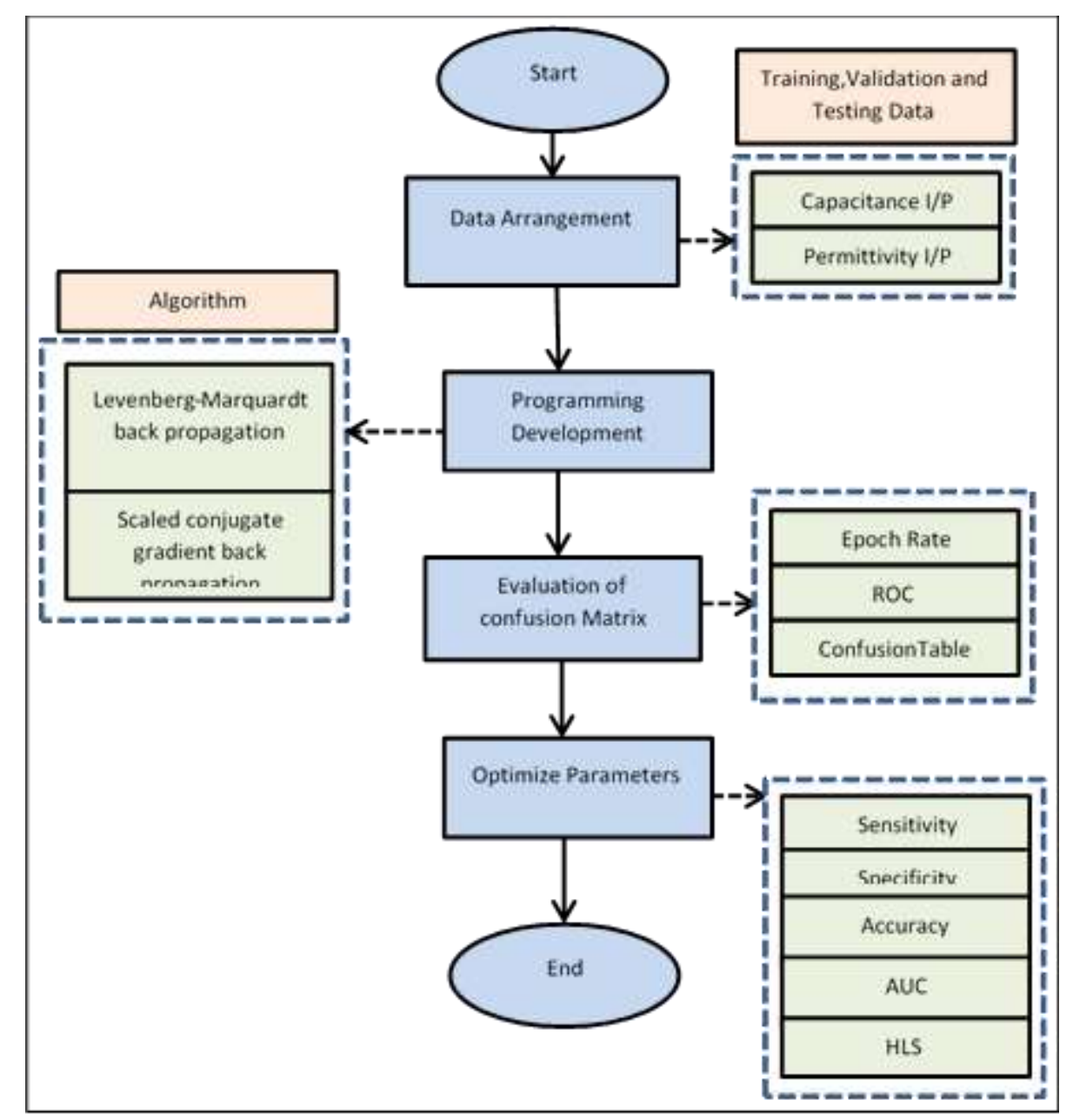

Figure 1. ANN modelling flow chart

Table 1. Number of datasets arranged for the use of neural network algorithm

\begin{tabular}{ccccc}
\hline Input Measurement & Training set & Testing set & Validation set & Total \\
\hline Permittivity & 420 & 90 & 90 & 600 \\
Capacitance & 420 & 90 & 90 & 600 \\
Total & 840 & 180 & 180 & 1200 \\
\hline
\end{tabular}

There were several evaluation parameters attained for the modelling. These evaluation parameters were used to obtain the best model. The evaluation parameters involved were hidden layer size, sensitivity, specificity, accuracy, and area under the curve (AUC). Programming based on LM and SCG algorithms was done using MATLAB R2017a software, which was developed by MathWorks. 


\section{RESULTS AND DISCUSSION}

After running all the experiments and statistical analysis, the readings to provide inputs for the ANN were established. This section provides the platform to develop an intelligence classifier with the use of input measurement from the experiment. In that section, the classification process involving data arrangement and optimisation of ANN model were discussed and suggested that measurements recommended as inputs to this classifier came from single input category. The explanation of the input category will be analysed next to explain the findings of the most optimised model based on the performance evaluation criteria that have the best hidden layer size, minimum MSE value, accuracy, area under the curve for ROC plot, sensitivity and specificity.

A systematic analysis of 24 models of performance measures was used in both LM and SCG for each measurement input model. Each model represents its performance measure with a set of changes in the hidden layer size, which was gradually increased from 3 to 49 with a step size of 2 each time of simulation. Based on the information, there will be 24 models to be generated from each measurement input category for LM and SCG. Only the best models from each input category from LM or SCG with the most optimised performance were selected. The sequence of finding the most optimised model based on the comparison of the input measurements between different models are shown in Table 2 .

Table 2. Sequence of finding the most optimised model

\begin{tabular}{ccccc}
\hline Input (I/P) & I/P Measurement & No. of & Algorithm & $\begin{array}{c}\text { No. of Optimised } \\
\text { Model }\end{array}$ \\
\hline Categories & Models & 24 & LM & 1 \\
\multirow{3}{*}{ Single I/P } & \multirow{2}{*}{ Relative Permittivity } & 24 & SCG & 1 \\
& & 24 & LM & 1 \\
& \multirow{2}{*}{ Capacitance } & 24 & SCG & 1 \\
\hline
\end{tabular}

The explanation of this section starts with the analysis of mean square error for the most optimised model selected from the single input category. This is followed by the analysis of the performance evaluation criteria based on the confusion matrix table. By comparing the performance evaluation criteria between LM and SCG algorithms, significant differences of measured optimised parameters were analysed and synthesised. The results and discussions in this section will show the most optimised model for the single input data. Table 3 shows the summary of the selected models for single input category.

Table 3. Summarised (MSE) analysis from single inputs

\begin{tabular}{|c|c|c|c|c|}
\hline Input & Measurement & Model & $\begin{array}{c}\text { Hidden Layer } \\
\text { Size }\end{array}$ & $\begin{array}{c}\text { Minimum MSE } \\
\text { value }\end{array}$ \\
\hline \multirow{2}{*}{ Single } & Relative Permittivity & ANN1IPHS27neu & 27 & after 28 epochs \\
\hline & Capacitance & ANN2IPHS7neu & 7 & after 13 epochs \\
\hline
\end{tabular}

From the result of each measurement input, it was found that models that have the lowest number of hidden layer among all optimised models were from the models ANN2IPHS7neu. Model ANN1IPHS27neu was chosen to be the most optimised model for a single input relative permittivity measurement followed by model ANN2IPHS7neu for a single input capacitance measurement. The fact that based on the mean square error (MSE) alone was not enough to prove that the selected most optimised models from LM and SCG were the best among all. This fact can be further reinforced by comparing the values of all measured parameters for optimisation. The optimisation for the best model could be best done based on the evaluation parameters and will be described in the next section of confusion matrix.

The entire selected models were based on its performance characteristics. This evaluation of performance would be best visualised using a confusion matrix. This matrix contains information about sensitivity, specificity, and accuracy to evaluate the best model. The correctness of a classification can be evaluated by computing the number of correctly recognised class [15-19]. These four counts constitute a confusion matrix that shows the evaluation information for the best model. In regards to the confusion matrix from all input measurements, the classification measurement values of the confusion matrix can be observed by referring to all data confusion matrix [20-22].

From Figure 2(a), for single input relative permittivity measurement, the selected optimised model from SCG had a value of sensitivity equal to $79.00 \%$ and specificity equal to $69.00 \%$ compared with the optimised model from LM, which had a value of sensitivity equal to $77.00 \%$ and specificity equal to $73.67 \%$. From Figure 2(b), for single input capacitance measurement, the selected optimised model from SCG had a 
value of sensitivity equal to $98.00 \%$ and specificity equal to $81.00 \%$ compared with the optimised model from LM, which had a value of sensitivity equal to $94.67 \%$ and specificity equal to $84.67 \%$.

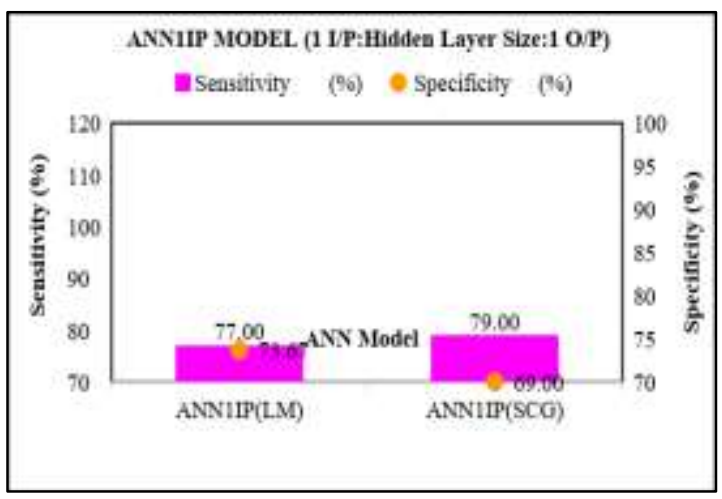

(a)

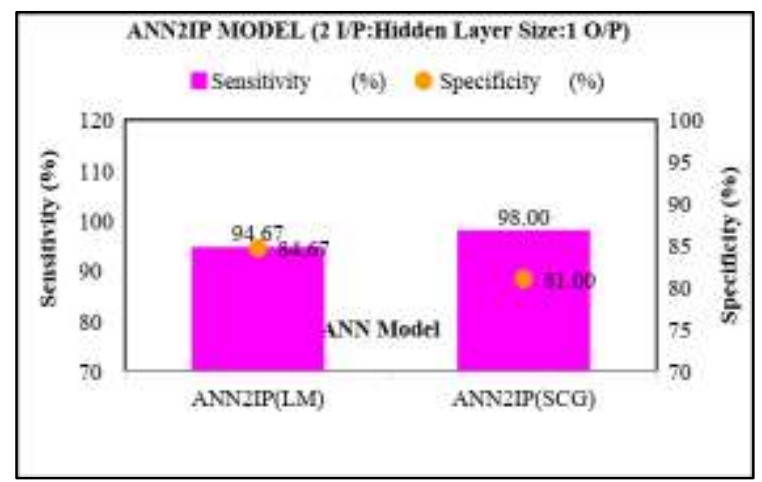

(b)

Figure 2. Comparisons of Sensitivity and Specificity for Single Input Categories, (a) Single input relative permittivity measurement, (b) Single input capacitance measurement

Accuracy and Area under Curve (AUC) are important factors in determining the best model in the ANN classification process and it has been proven that these two factors have the ability in determining the measurement for evaluating the best model [23-25]. The evaluation factors contributing to the performance of the selected models were further discussed towards accuracy and AUC. For the process of obtaining the value of AUC, the Receiver Operating Characteristic (ROC) curves need to be carried out first. The ROC curve is another parameter that needs to be evaluated before any conclusive decision in selecting the most optimised model. The parameters in ROC plot that need to be analysed are the Euclidean Distance (ED) and Bookmarker Informedness (BM) for determining the best threshold (i.e. closest indicate to the perfect point $(0,1)$ in the ROC plot) and the total area under curve (AUC). Based on the observation from Figure 3, ROC curve for all models lies above the diagonal line. Selection for the best model between LM and SCG algorithm are now at the last criteria evaluation, which describes the results from calculating the Hidden Layer Size (HLS) and Area under Curve (AUC).

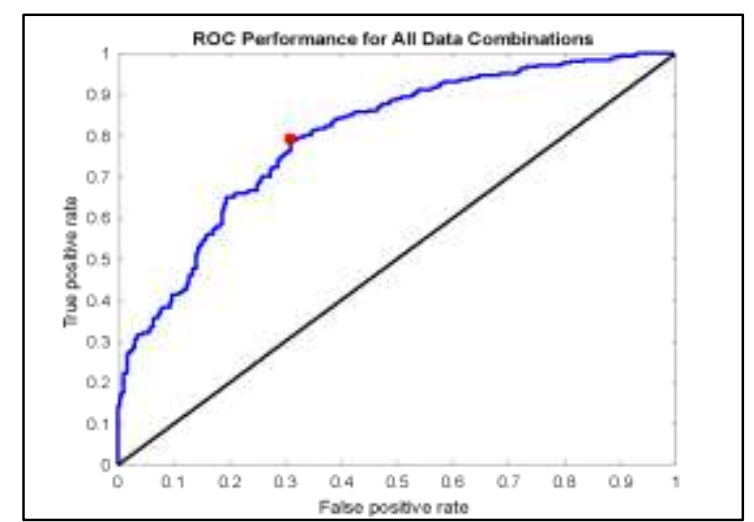

(a)

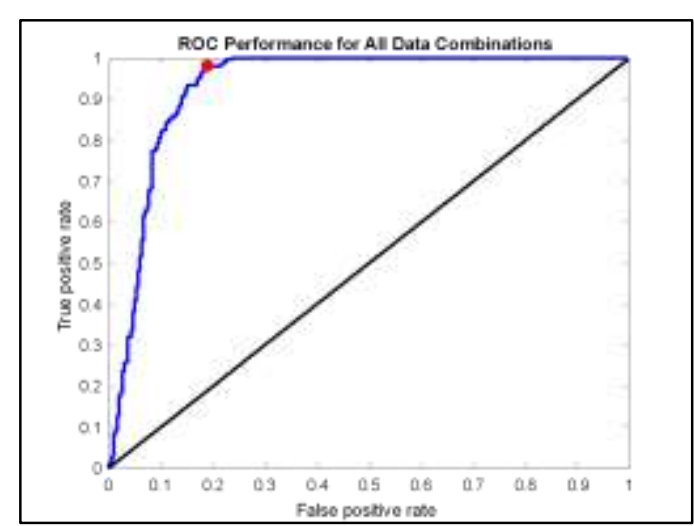

(b)

Figure 3. ROC curve plot for the selected optimised model for all input categories, (a) Relative permittivity (SCG), (b) Capacitance (SCG)

The optimisation process was start with the calculation of HLS involved in the model. Table 4 shows the HLS obtained from every optimised model calculated. The reason of using HLS in the optimisation process lies in the theory that claims when lower connection is obtained, the better the system would be and this would definitely strengthens the fact in choosing the best model [26, 27]. 
Table 4. Hidden layer size for all optimised models

\begin{tabular}{ccccccc}
\hline $\begin{array}{c}\text { Input } \\
\text { Categories }\end{array}$ & Algorithm & HLS & Sensitivity (\%) & Specificity (\%) & Accuracy (\%) & AUC (\%) \\
\hline \multirow{3}{*}{ Single } & LM & 49 & 77.00 & 73.67 & 75.33 & 80.99 \\
& SCG & 27 & 79.00 & 69.00 & 74.00 & 79.31 \\
& LM & 9 & 94.67 & 84.67 & 89.67 & 93.09 \\
& SCG & 7 & 98.00 & 81.00 & 89.50 & 92.54 \\
\hline
\end{tabular}

The consideration of an optimised model were further analysed by looking at the best HLS between each model from each input category. Starting from the first model in single input relative permittivity measurement, the selected optimised model was from SCG, which had a value of HLS equal to 27 compared with the optimised model from LM, which had a value of HLS equal to 49. In the single input capacitance measurement, the selected optimised model was from SCG, which had a value of HLS equal to 7 compared with the optimised model from LM, which had a value of HLS equal to 9. The selection that was based on the evaluation criteria can be summarised by looking at the lowest number of hidden layer size, the highest sensitivity and specificity, the optimum value of accuracy and the highest AUC among the optimised models. The summary of the selected models can be shown in Table 5.

Table 5. The summary of performance evaluation parameters results for the selected optimised model

\begin{tabular}{cccccccc}
\hline \multirow{2}{*}{ Input } & $\begin{array}{c}\text { Ann Model } \\
\text { Structure }\end{array}$ & HLS & $\begin{array}{c}\text { Sensitivity } \\
(\%)\end{array}$ & $\begin{array}{c}\text { Specificity } \\
(\%)\end{array}$ & $\begin{array}{c}\text { Accuracy } \\
(\%)\end{array}$ & AUC $(\%)$ & Net Name \\
\hline \multirow{5}{*}{ Single } & ANN1IP(LM) & 49 & 77.00 & 73.67 & 75.33 & 80.99 & \\
& ANN1IP(SCG) & 27 & 79.00 & 69.00 & 74.00 & 79.31 & ANN1IPHS27neu \\
& ANN2IP(LM) & 9 & 94.67 & 84.67 & 89.67 & 93.09 & \\
& ANN2IP(SCG) & 7 & 98.00 & 81.00 & 89.50 & 92.54 & ANN2IPHS7neu \\
\hline
\end{tabular}

\section{CONCLUSION}

In conclusion, it shows that the developed model for classifying between healthy trees and white root disease infected trees based on electrical properties is reliable. This was based on the overall observation of the performance evaluation parameters where the selected most optimised model from each input category can be proven to be significant. These were based on the comparison between each of the performance evaluation parameter result including the HLS. Two types of models have been selected throughout the process of selecting the most optimised model based on the performance evaluation parameter results and these selected optimised models are ANN1IP(SCG) and ANN2IP(SCG).

\section{AKNOWLEDGEMENT}

The author would like to thank Universiti Teknologi MARA, Cawangan Pulau Pinang, Kampus Permatang Pauh for financially supporting this research.

\section{REFERENCES}

[1] R. E. Uhrig, "Introduction to artificial neural networks," in Industrial Electronics, Control, and Instrumentation, 1995, Proceedings of the 1995 IEEE IECON 21st International Conference on, vol. 1, pp. 33-37, 1995.

[2] P. K. Simpson, "Fuzzy min-max neural networks - Part 2: Clustering," IEEE Transactions on Fuzzy Systems, vol. 1, no. 1, p. 32, 1993.

[3] P. Daponte and D. Grimaldi, "Artificial neural networks in measurements," Measurement, vol. 23, no. 2, pp. 93115, 1998.

[4] P. G. Bartley, et al., "Permittivity determination by using an artificial neural network," in IMTC/99. Proceedings of the 16th IEEE Instrumentation and Measurement Technology Conference (Cat. No.99CH36309), vol. 1, pp. 27-30, 1999.

[5] C. Qian, et al., "The Measurement for Permittivity of Materials Based on Artificial Nerve Network," pp. 272-274, 2011.

[6] A. Hasan and A. F. Peterson, "Measurement of Complex Permittivity using Artificial Neural Networks," IEEE Antennas and Propagation Magazine, vol. 53, no. 1, pp. 200-203, 2011.

[7] H. Hashim, et al., "An Intelligent Classification Model for Rubber Seed Clones Based on Shape Features through Imaging Techniques," in 2010 International Conference on Intelligent Systems, Modelling and Simulation, pp. 2531, 2010.

[8] P. S. Prerana, "Comparative Study of GD, LM and SCG Method of Neural Network for Thyroid Disease Diagnosis,” International Journal of Applied Research, vol. 1, no. 10, pp. 34-39, 2015. 
[9] A. S. Agustin and S. S. Babu, "Thyroid Classification as Normal and Abnormal using SCG based Feed Forward Back Propagation Neural Network Algorithm," 2013.

[10] Y. Sewsynker-Sukai, et al., "Artificial neural networks: an efficient tool for modelling and optimization of biofuel production (a mini review)," Biotechnology \& Biotechnological Equipment, vol. 31, no. 2, pp. 221-235, 2017.

[11] H. Karim, et al., "Comparison of Neural Network Training Algorithms for Classification of Heart Diseases," 2018.

[12] K. G. Adi, et al., "Analysis and Detection of Cholesterol by Wavelets based and ANN Classification," Procedia Materials Science, vol. 10, pp. 409-418, 2015.

[13] N. Messikh, et al., "The use of a multilayer perceptron (MLP) for modelling the phenol removal by emulsion liquid membrane," Journal of Environmental Chemical Engineering, vol. 5, no. 4, pp. 3483-3489, 2017.

[14] Z. Ye and M. K. Kim, "Predicting Electricity Consumption in a Building Using an Optimized Back-propagation and Levenberg-Marquardt Back-propagation Neural Network: Case Study of a Shopping Mall in China," Sustainable Cities and Society.

[15] D. Wackerly, et al., "Mathematical statistics with applications," Cengage Learning, 2014.

[16] C. D. Richmond and M. I. O. T. L. L. LAB, "Mean Squared Error Performance Prediction of Maximum-Likelihood Signal Parameter Estimation," massachusetts inst of tech lexington lincoln lab, 2003.

[17] N. Pentreath, "Machine Learning with Spark," Packt Publishing, 2015.

[18] M. Sokolova and G. Lapalme, "A systematic analysis of performance measures for classification tasks," Information Processing \& Management, vol. 45, no. 4, pp. 427-437, 2009.

[19] T. S. Gunawan, et al., "Artificial Neural Network Based Fast Edge Detection Algorithm for MRI Medical Images," Indonesian Journal of Electrical Engineering and Computer Science, vol. 7, no. 1, pp. 123-130, 2017.

[20] A. F. M. Sampian, et al., "Vision system for detection of white root disease infection based on capacitance properties," in Automatic Control and Intelligent Systems (I2CACIS), IEEE International Conference on, pp. 186$189,2016$.

[21] M. Mohamed, et al., "Feature extraction of speech signal and heartbeat detection in angry emotion identification," International Journal of Computer Science and Electronics Engineering (IJCSEE), vol. 1, no. 1, 2013.

[22] T. S. Gunawan and M. Kartiwi, "On the comparison of line spectral frequencies and mel-frequency cepstral coefficients using feedforward neural network for language identification," Indonesian Journal of Electrical Engineering and Computer Science, vol. 10, no. 1, pp. 168-175, 2018.

[23] H. Jin and C. X. Ling, "Using AUC and accuracy in evaluating learning algorithms," IEEE Transactions on Knowledge and Data Engineering, vol. 17, no. 3, pp. 299-310, 2005.

[24] W. Aziz, et al., "An accurate pattern classification for empty fruit bunch (EFB) based on the age profile of oil palm tree using neural network," International Journal of Electrical \& Computer Engineering, vol. 9, 2019.

[25] A. W. Setiawan, et al., "Classification of palm oil fresh fruit bunch using multiband optical sensors," International Journal of Electrical \& Computer Engineering, vol. 9, 2019.

[26] J. D. Olden, et al., "An accurate comparison of methods for quantifying variable importance in artificial neural networks using simulated data," Ecological Modelling, vol. 178, no. 3, pp. 389-397, 2004.

[27] P. J. Das, et al., "Chapter 13 - Artificial Neural Network as Helping Tool for Drug Formulation and Drug Administration Strategies," in M. Puri, et al. Editors, Artificial Neural Network for Drug Design, Delivery and Disposition, Boston: Academic Press, pp. 263-276, 2016. 